\title{
The Gaussian beam mode analysis of off-axis aberrations in long wavelength optical systems
}

\author{
T.J. Finn ${ }^{\mathrm{a}, *}$, N. Trappe ${ }^{\mathrm{a}}$, J.A. Murphy ${ }^{\mathrm{a}}$, S. Withington ${ }^{\mathrm{b}}$ \\ a Experimental Physics Department, National University of Ireland, Maynooth, Kildare, Ireland \\ ${ }^{\mathrm{b}}$ Cavendish Laboratory, Madingley Road, Cambridge CB3 OHE, UK
}

Received 1 September 2007

Available online 6 November 2007

\begin{abstract}
An issue of major concern in the design of long wavelength systems is optical aberration or distortion, which can be particularly severe in off-axis systems. Aberrations occur in both lenses and mirrors and in this paper we present a novel method capable of modelling offaxis mirror configurations. Aberrations degrade fundamental receiver coupling coefficients such as aperture efficiency while increasing spillover power losses. For single pixel instruments this will lead to much longer integration times and the possibility of stray light. For imaging arrays the aberrations cause a departure from perfect point imaging by increasing coupling to array detectors located at angles further off the bore sight of the telescope. This paper verifies a matrix-based scheme using Gaussian beam mode analysis (GBMA) for predicting aberrations from off-axis mirrors. The applied technique was originally described in (S. Withington, A. Murphy, G. Isaak, Representation of mirrors in beam waveguides as inclined phase transforming surfaces, Infrared Phys. Tech. 36(3) (1995) 723-734. [1]) and in this paper we exploit the theory and validate the approach with a series of examples using off-axis conic sections. We present the predictions for both a fundamental Gaussian and a scalar horn field illuminating various off-axis mirror configurations including different angles of incidence. A commercially available physical optics (PO) software package, GRASP8 ${ }^{\text {TM }}$, is used to validate the accuracy of these scalar GBMA predictions.
\end{abstract}

(C) 2007 Elsevier B.V. All rights reserved.

PACS: 41.20.Jb; 07.57.Ty; 42.25.Fx

Keywords: Aberrations; Long wavelength; Quasioptics (QO); Gaussian beam modes (GBM); Physical optics

\section{Introduction}

In [1] a comprehensive derivation of the theoretical approach is outlined without subsequent application of the technique to submillimetre optical systems due to the limited computational resources available at the time of writing. In this paper we summarise the method and apply the technique to a number of relevant examples where both phase and amplitude distortion are important and illustrate the accuracy of the technique. The approach is based on treating the off-axis mirror as an inclined phase transform-

\footnotetext{
* Corresponding author.

E-mail address: t.finn@oan.es (T.J. Finn).

$U R L:$ http://www.physics.nuim.ie (T.J. Finn).
}

ing surface which is considered to be the aberrating thin lens equivalent of the mirror (i.e. the systems are considered physically to be in transmission rather than in reflection). The scatter matrix technique determines the power scattered from a propagating mode set incident on the input side of the inclined surface to a mode set on the output side of the surface which may then be propagated to any desired plane where the aberrated field may be reconstructed. The scattering matrix is evaluated by considering the diffractive spreading of the beam within the volume of the mirror. This manifests itself as a variation of the beam width and phase radius curvature of the beam across the mirror surface related to the depth of the mirror as a function of position. In general GBMA ignores this variation since the surface is considered to be normal to the 
propagating beam and thus there is only one representative beam width at any plane. The matrix is determined by the overlap integral between the input and output mode sets which are characterised by GBM parameters.

The fact that the input and output mode sets are not orthogonal over the mirror surface must also be included in the inclined phase transforming surface technique. Any GBM calculation is only valid for such mode sets. In the approach adopted in this paper the input and output planes are tilted relative to each other and thus a further scattering matrix must be evaluated to correct for this incomplete description. The matrix is determined by an appropriate overlap integral between the input and output mode sets described below. By combining the two scattering matrices accounting for the mirror shape and the non-orthogonality of the modes a final matrix can be determined which fully describes the aberrating properties of the off-axis mirror within the limitations of this paraxial, scalar approach. The shapes of the conic surfaces are described by a series of geometrical relationships [1].

A majority of long wavelength quasioptical systems are designed utilising ray-tracing and fundamental Gaussian beam mode analysis which ignore the departure from perfect imaging that arises from off-axis rays and curvature mismatches between the mirror surface and the incoming phase front caused by the diffractive, long wavelength nature of a propagating beam. As well as phase front errors, aberrations also arise from bandwidth usage effects. In a modal description this results in the scattering of power amongst modes thus requiring a multi-moded analysis. The mode set used to describe such a field as it propagates limited by the paraxial and scalar assumptions are comprehensively described in [2-4]. If waveguide modes are to be included in the analysis a scattering matrix technique, based on a mode matching approach adopted in [5] and previously used to predict the field radiated by horn antennas is described in term of TE and TM modes. These modes can be transformed to freespace as illustrated in $[6,7]$.

\section{Theory}

In the modal view of cylindrically symmetric free space propagation, the modes can be represented by associated Laguerre-Gaussian polynomials. In the case of asymmetric or Cartesian systems, Hermite-Gaussian polynomials are the preferred choice of mode set. The higher order Gaussian beam solutions are given in [4]

$$
\begin{aligned}
\Psi_{m n}(x, y)= & h_{m}\left(\frac{\sqrt{2} x}{W}\right) h_{n}\left(\frac{\sqrt{2} y}{W}\right) \exp \left[-\mathrm{j} k\left(z+\frac{r^{2}}{2 R}\right)\right] \\
& \exp \left[\mathrm{j} \phi_{m n}\right],
\end{aligned}
$$

where $h_{m}$ and $h_{n}$ are normalised Hermite function of the form

$$
\begin{aligned}
& h_{m}\left(\frac{\sqrt{2} x}{W}\right)=\frac{1}{\sqrt{2^{m-\frac{1}{2}} m ! \sqrt{\pi W^{2}}}} H_{m}\left(\sqrt{2} \frac{x}{W}\right) \exp \left[-\frac{x^{2}}{W^{2}}\right], \\
& h_{n}\left(\frac{\sqrt{2} y}{W}\right)=\frac{1}{\sqrt{2^{n-\frac{1}{2}} n ! \sqrt{\pi W^{2}}}} H_{n}\left(\sqrt{2} \frac{y}{W}\right) \exp \left[-\frac{y^{2}}{W^{2}}\right],
\end{aligned}
$$

where $W$ is the beam width, $R$ is the phase radius of curvature and $\phi$ is the phase slippage of each mode. The appropriate normalizing factors are assumed. The electric field can be calculated at any plane by a linear summation of modes and mode coefficients (i.e. $\sum_{m} A_{m} \Psi_{m}$ ). The coefficients determine the amount of power contained in each mode. Only the phase slippage is mode dependent and the classical ABCD matrix method adapted for GBM propagation is used throughout this paper to track the evolution of the GBM parameters through freespace $[2,4,8]$.

\subsection{Aberrations from off-axis mirrors}

In order to account for the aberrating effects of a mirror used in an off-axis configuration, [1] has proposed treating mirrors as inclined phase transformers where the variation of the GBM parameters of beam width $W$, phase radius of curvature $R$, and the modal phase slippage are considered. The input mode coefficients are scattered into the output mode coefficients by considering two types of scattering. Firstly, the scattering due to the two mode sets not being orthogonal ( $\beta$ matrix) and secondly the actual amplitude and phase distortion added across the mirror surface $(\alpha$ matrix). For the non-orthogonally generally GBM are only considered complete sets when they are orthogonal otherwise corrections must be made [9]. This is given by [1]

$$
\begin{aligned}
\beta_{i j r s}= & \int_{-\infty}^{\infty} h_{r}\left(\frac{\sqrt{2 x_{0}}}{w_{\text {mirror }}}\right) h_{s}\left(\frac{\sqrt{2 y_{0}}}{w_{\text {mirror }}}\right) h_{i}\left(\frac{\sqrt{2 x_{0}}}{w_{\text {mirror }}}\right) h_{j}\left(\frac{\sqrt{2 y_{0}}}{w_{\text {mirror }}}\right) \\
& \exp \left[\mathrm{j}(r-i) \varphi_{i, r}\left(x_{0}, y_{0}\right)\right] \mathrm{d} x \mathrm{~d} y \delta_{j, s},
\end{aligned}
$$

where $W_{\text {mirror }}$ is the size of the beam width at a location ( $x$, $y$ ) and $\varphi_{m n}$ is the phase slippage at a point $(x, y)$. The value of $W_{\text {mirror }}$ will vary across the mirror surface from a central point, which corresponds to the chief ray intercept from a geometrical perspective. Prior to this we have always explicitly assumed that the value of $W$ did not vary across the mirror surface. Fig. 1a and b illustrates the geometry of the problem where the mirror is tilted by an angle $\theta . \Delta z$ is the thickness of the mirror, $x$ and $z$ are the original coordinate system and $x^{\prime}$ and $z^{\prime}$ represent the tilted coordinate system.

Secondly, the actual amplitude distortion added across the mirror surface is dependent on the variation of not only $W$ but also the phase radius of curvature $R$. From the geometry of the conic section (elliptical or parabolic) in question it is possible to relate the depth of the mirror to the beam variation over the surface as illustrated below. The scattering matrix between input and output mode set is given in [1] as 


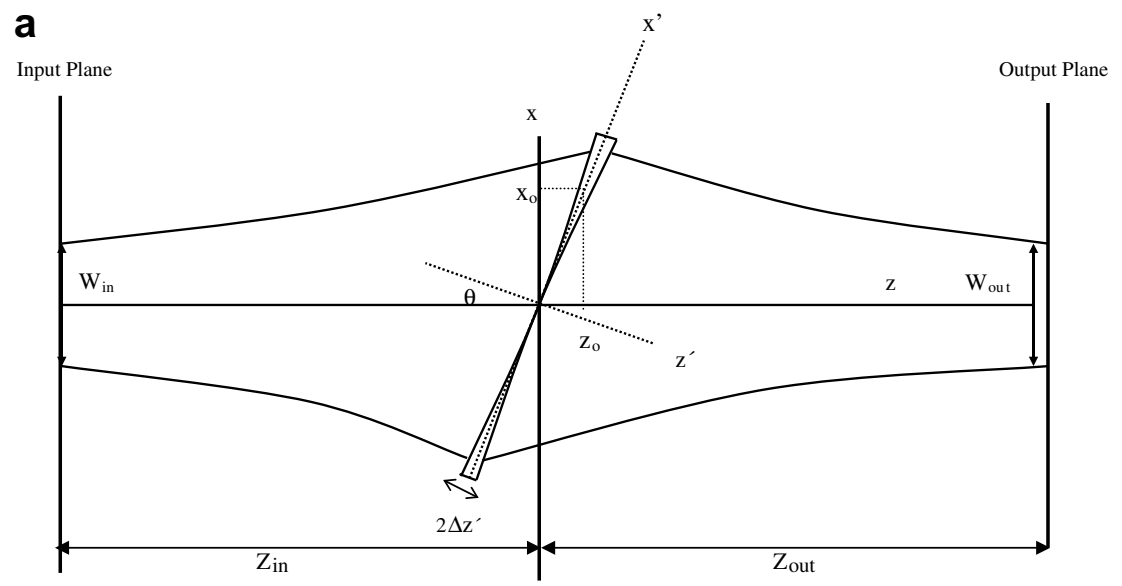

b

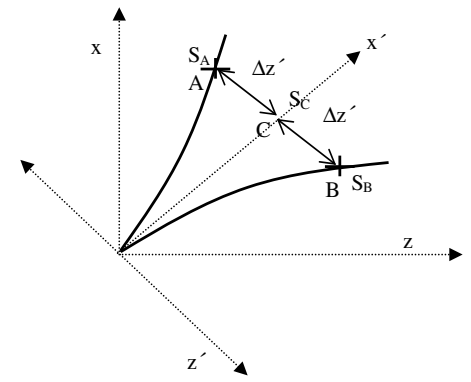

Fig. 1. The geometry of the thin lens approximation of an off-axis mirror.

$$
\begin{aligned}
\alpha_{i j m n}= & \int_{-\infty}^{\infty} h_{m}\left(\frac{\sqrt{2 x_{0}}}{w_{1}}\right) h_{i}\left(\frac{\sqrt{2 y_{0}}}{w_{2}}\right) \exp \left[\mathrm{j}(m+n+1) \varphi_{\text {in }}\right] \exp \left[-\mathrm{j}(i+j+1) \varphi_{\text {out }}\right] \\
& \times \int_{-\infty}^{\infty} h_{n}\left(\frac{\sqrt{2 x_{0}}}{w_{1}}\right) h_{j}\left(\frac{\sqrt{2 y_{0}}}{w_{2}}\right) \exp \left[-\mathrm{j} k \frac{\left(\left(x_{0}+\Delta x\right)^{2}+y_{0}^{2}\right)}{R_{1}\left(z_{1}-\Delta z\right)+\sqrt{R_{1}\left(z_{1}-\Delta z\right)^{2}+\left(\left(x_{0}+\Delta x\right)^{2}+y_{0}^{2}\right)}}\right] \\
& \times \exp \left[+\mathrm{j} k \frac{\left(\left(x_{0}-\Delta x\right)^{2}+y_{0}^{2}\right)}{R_{2}\left(z_{2}+\Delta z\right)-\sqrt{R_{2}\left(z_{2}+\Delta z\right)^{2}+\left(\left(x_{0}-\Delta x\right)^{2}+y_{0}^{2}\right)}}\right] \exp \left[\mathrm{j} k 2 \Delta z^{\prime} \cos \theta\right] \mathrm{d} y_{0} \mathrm{~d} x_{0},
\end{aligned}
$$

where $\Delta z$ is the thickness of the mirror and $R_{1}$ and $R_{2}$ are the incident and output radius of curvature before and after the transformation by the mirror surface. Using the ABCD matrix method the evolution the input beam parameters $W, R$ and $\varphi$ can be calculated as a function of position across the mirror surface. These values can then be used to calculate $\alpha$ and $\beta$ matrices to determine the full scattering matrix for a mirror. The integral is carried out over the surface of the mirror and so allows the physical size of the mirror or edge taper to be included. A sequence of mirrors can be analysed by using the output of one mirror as the input for the next. The overall scattering matrix for a mirror is a combination of $\alpha$ and $\beta$ and can be calculated by

$S_{m n}=\beta_{m n}^{-1} \alpha_{m n}$,

and the output mode coefficients are calculated by

$B_{n}=\sum_{m} S_{m n} A_{m}$.

This allows the prediction of the amplitude and phase distortion introduced by an off-axis mirror. Additionally any field input that is desired to illuminate the system can easily be adopted in this framework.

\section{Examples}

In this section we introduce different examples where different geometries and different configurations of mirrors are illustrated with the technique. These examples include an elliptical and parabolic mirror, each illuminated by a Gaussian input, and also an elliptical mirror configuration making a wavelength independent Gaussian beam telescope is investigated. In each case the equivalent output beam is predicted by a well-established physical optics technique using GRASP8 ${ }^{\mathrm{TM}}$, a recognised bench mark package used to validate and compare the accuracy of the approach illustrated here.

\subsection{Elliptical mirror with Gaussian input beam}

Initially we will consider a fundamental Gaussian illuminating an off-axis elliptical mirror. Elliptical mirrors typically find use in QO systems to transform an input radius of curvature, $R_{\text {in }}$ to a desired output radius of curvature, $R_{\text {out }}$ where $R_{1}=R_{\text {in }}$ and $R_{2}=R_{\text {out }}$ (Fig. 4). In this scalar approximation the fraction of power that is contained in the fundamental mode of the reflected beam is found from [2] to be 


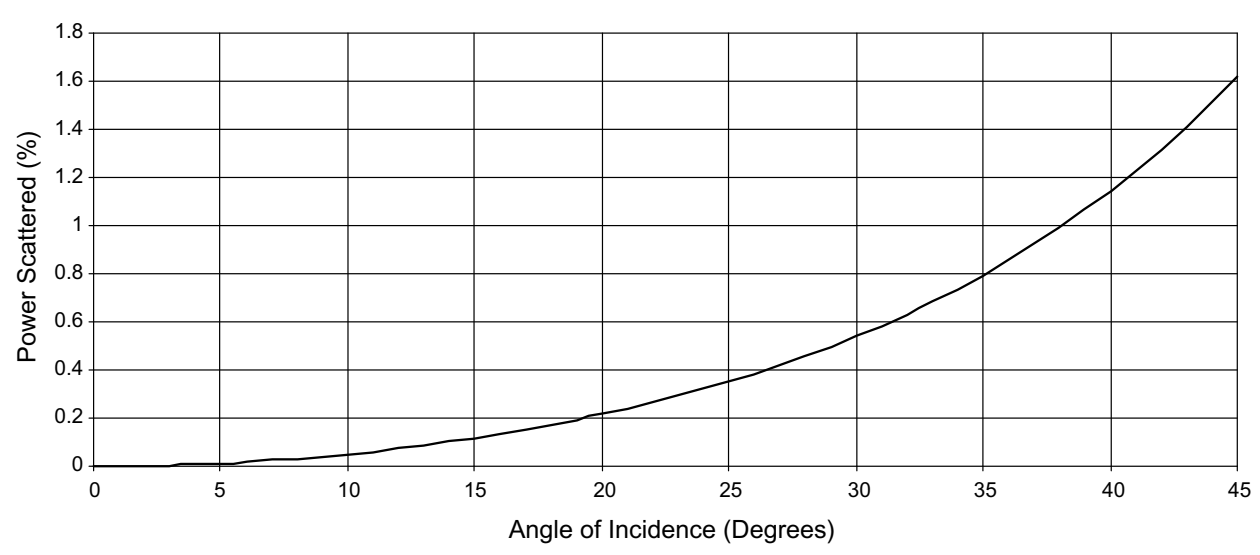

Fig. 2. The relationship of the amount of power removed from the fundamental mode to higher order modes as a function of incidence angle for an offaxis mirror with a fixed beam width and focal length.

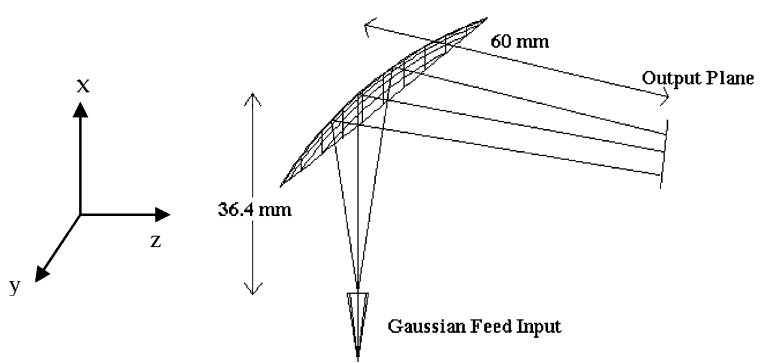

Fig. 3. The geometry of the elliptical mirror configuration [plot generated from GRASP GUI].

$K_{f}=1-U^{2}=1-\left(\frac{W_{m} \tan \left[\theta_{i}\right]}{2 \sqrt{2} f}\right)^{2}$,

where $f$ is the effective focal length of the ellipse/paraboloid. It is evident that if one considers a mirror of fixed focal length and with a fixed beam width at its surface then the amount of power scattering that occurs depends on the angle of incidence. Fig. 2 illustrates the behaviour of the power scattering from the fundamental mode (related to level of aberration), which has an asymptotic form.

Physically lower Gaussicity relates to the larger mismatch between the mirrors curved surface and the incoming field amplitude distribution. This is better matched at lower angles of incidence when the mirror is more "faceon" to the incoming field. The ability of the GBM approach to model this effect will now be investigated for angles of incidence of $2.5^{\circ}, 5^{\circ}, 10^{\circ}, 20^{\circ}, 30^{\circ}, 40^{\circ}$, and $45^{\circ}$. In this initial example the input is a Gaussian beam waist with $W=1.23 \mathrm{~mm}(R=\infty)$ at a frequency of $480 \mathrm{GHz}$ located at a distance of $36.4 \mathrm{~mm}$ on front of the elliptical mirror with a focal length of $30 \mathrm{~mm}$ as illustrated in Fig. 3. The mirror diameter is $4 W$. For this particular configuration the angle of incidence is $30^{\circ}$.

For the evaluation of the $\alpha$ matrix a critical parameter is the mirror thickness $\Delta z$ or alternatively its height above the tangent plane, which is dependent on the mirror geometry.

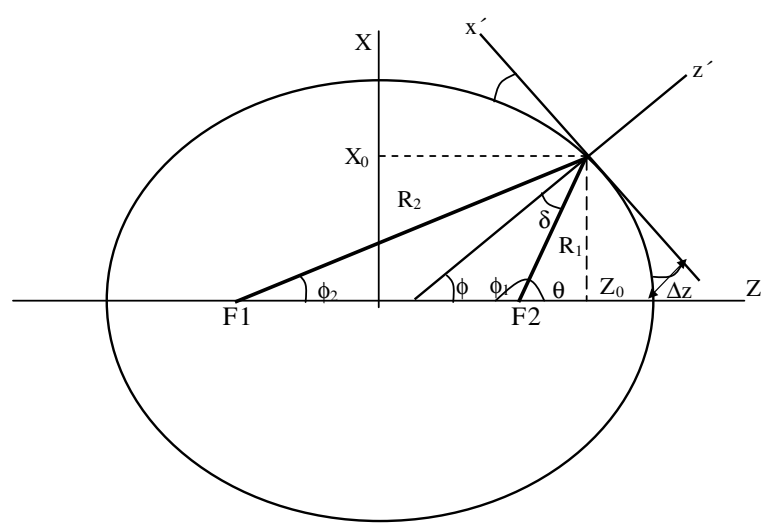

Fig. 4. The geometry of an off-axis elliptical mirror [1].

To determine this and analyse the ellipsoid we consider the geometry in Fig. 4. The major and minor axes are given by

$a=\frac{R_{1}+R_{2}}{2}, \quad b=\sqrt{R_{1} R_{2}} \cos \delta$.

The angle that the normal to the tangent plane makes relative to the axis of rotation is given by [1]

$\tan \phi_{1}=\frac{\sin (2 \delta)}{\left[\frac{R_{1}}{R_{2}}-\cos (2 \delta)\right]}$,

and from the geometry we can say that $\phi=\pi-\phi_{1}-\delta$ and $\theta=\pi-\phi_{1}$. The intercept of the mirror is located at

$X_{0}=R_{1} \sin (\theta)$ and $Z_{0}=R_{1} \cos (\theta)+a e$,

where $e$ is the eccentricity.

Again from [1] it is shown that by shifting and rotating the $(x, z)$ coordinate frame the thickness of the mirror surface is then given by

$\Delta z^{\prime}=\left|\frac{-B+\sqrt{B^{2}-4 A C}}{2 A}\right|$, 
Table 1

The associated parameters for the elliptical mirror

\begin{tabular}{ll}
\hline Elliptical mirror input parameters & \\
\hline Wavelength & $0.625 \mathrm{~mm}$ \\
$W_{\text {in }}$ & $1.23 \mathrm{~mm}$ \\
Input distance & $36.4 \mathrm{~mm}$ \\
Output distance & $60 \mathrm{~mm}$ \\
Focal length $f$ & $30 \mathrm{~mm}$ \\
$a$ of the ellipse & $60 \mathrm{~mm}$ \\
$b$ of the ellipse & $56.38 \mathrm{~mm}$ \\
$R_{1}$ & $60 \mathrm{~mm}$ \\
$R_{2}$ & $60 \mathrm{~mm}$ \\
$\delta$ & $30^{\circ}$ \\
\hline
\end{tabular}

where

$$
\begin{aligned}
A= & \frac{\sin ^{2} \phi}{b^{2}}+\frac{\cos ^{2} \phi}{a^{2}} \\
B= & \frac{2 x_{0} \cos \phi \sin \phi}{\cos \delta}\left(\frac{1}{b^{2}}-\frac{1}{a^{2}}\right)+\frac{2 X_{0} \sin \phi}{b^{2}}+\frac{2 Z_{0} \cos \phi}{a^{2}}, \\
C= & \frac{y_{0}^{2}}{b^{2}}+\left(\frac{x_{0}}{\cos \delta}\right)^{2}\left(\frac{\cos ^{2} \phi}{b^{2}}+\frac{\sin ^{2} \phi}{a^{2}}\right) \\
& +2\left(\frac{x_{0}}{\cos \delta}\right)\left(\frac{\cos \phi X_{0}}{b^{2}}-\frac{\sin \phi Z_{0}}{a^{2}}\right) .
\end{aligned}
$$

These parameters then have to be calculated for a specific mirror. For the considered mirrors the associated parameters are displayed in Table 1 for an angle of incidence of $30^{\circ}$.

The beam width at the mirror is $W_{\text {mirror }}=9 \mathrm{~mm}$, the output beam at $60 \mathrm{~mm}$ from the mirror has a beam width $W=4.1 \mathrm{~mm}$. Fig. 5 compares the GBMA theory with the equivalent physical optics output as the angle of incidence is increased and as expected the distortion increases. The equivalent field as predicted by GRASP ${ }^{\mathrm{TM}}$ is also shown below and it can be seen that the shift in peak intensity is well matched for all angles. Additionally good agreement is found with the GBMA technique down to $-40 \mathrm{~dB}$ for incident angles of $40^{\circ}$ or less and agreement to $-25 \mathrm{~dB}$ for a $45^{\circ}$ angle of incidence. This is mainly due to the fact that as the incidence angle increases more power is scattered into cross-polar components and GBMA is a scalar theory. The scalar approximation will limit the accuracy of the GBMA predictions, particularly further off-axis. Nevertheless excellent agreement can be observed even for an extreme example such as a full angle throw of $90^{\circ}$. Additionally a limited mode set of $100 \mathrm{Her}-$ mite modes is employed. An increase from 100 modes would improve agreement further off-axis but would result in much longer calculation times for a single mirror whereas 100 modes yields good agreement down to between -25 and $-40 \mathrm{~dB}$ which is sufficient for most applications.

Table 2 illustrates how the increasing off-axis angle leads to a decrease in Gaussicity from away from ideal optics. This is calculated by evaluating the overlap integral between the output field with its equivalent, on-axis, ideal, fundamental Gaussian field. As with Fig. 2 the trend of slowly increasing power scattering out of the fundamental up to $20-30^{\circ}$ followed by a sharp increase for higher angles can be observed. Also shown in Fig. 6 is the power contained in the fundamental mode as calculated from the scattering matrix technique. The predictions of the fundamental power scattering of the GBMA in comparison to that of Eq. (7) are also compared in Fig. 6 and exhibits good agreement in the general trend although the GBM technique predicts higher power scattering.

\subsection{Corrugated horn input to multi-mirror systems}

While the previous example is useful in highlighting the effect of increasingly off-axis angles on the beam quality and the accuracy of the GBMA technique, many QO feeds radiate beams that contain a significant amount of power in higher order modes. Additionally most QO systems will consist of more than one mirror. Thus in this section we demonstrate a corrugated horn input illuminating a twomirror Gaussian beam telescope. This configuration can be seen in Fig. 7 and images one horn aperture to another in a frequency independent manner. The corrugated horn was operated at $480 \mathrm{GHz}$ and is characterised by the following parameters: aperture diameter $=2.5 \mathrm{~mm}, W_{\text {aper }}=$ $0.6345 *$ aperture diameter and $R_{\text {aper }}=15.4 \mathrm{~mm}$.

Thus this is the multi-moded input equivalent of the previous example since the horn aperture is located $30 \mathrm{~mm}$ from the first off-axis elliptical mirror but the phase centre is located $6.4 \mathrm{~mm}$ behind the aperture and has a beam waist width $W=1.23 \mathrm{~mm}$. The mirror diameters are $4 W$. As with the previous example GRASP shall be used to validate the GBMA technique. These mirrors will introduce aberrations to the propagating beam that will alter the level of coupling to the symmetrical field of the second horn antenna. The input beam is a truncated Bessel model of a corrugated horn. The focal lengths are $f_{1}=f_{2}=30 \mathrm{~mm}$ and the input distance $d_{\text {in }}=30 \mathrm{~mm}$ and is equal to the output distance $d_{\text {out }}$. Finally, we shall also consider the accuracy of the phase predictions of GBMA.

This asymmetric arrangement of elliptical mirrors is a compensating configuration that can be derived from calculating the Gaussicity of the output of mirror 1 in comparison to mirror 2. The output field of mirror 1 at the plane of mirror 2 exhibits a Gaussicity of $94.8 \%$ and the output of mirror 2 at the second horn aperture plane a Gaussicity of $96.5 \%$ an improvement of $1.7 \%$. (The ideal field would exhibit a Gaussicity of 98\%.)

The field at the output plane is shown in Fig. 8 as predicted by GBM and by GRASP. The two methods exhibit excellent agreement down to $-25 \mathrm{~dB}$ in the $\mathrm{E}$ and $\mathrm{H}$ planes. Beyond this point GRASP predicts a narrower main beam but again this is due to the limiting assumptions made in the derivation of the GBM theory. The unaberrated $\mathrm{H}$ plane still clearly reproduces the horn input, as it should 

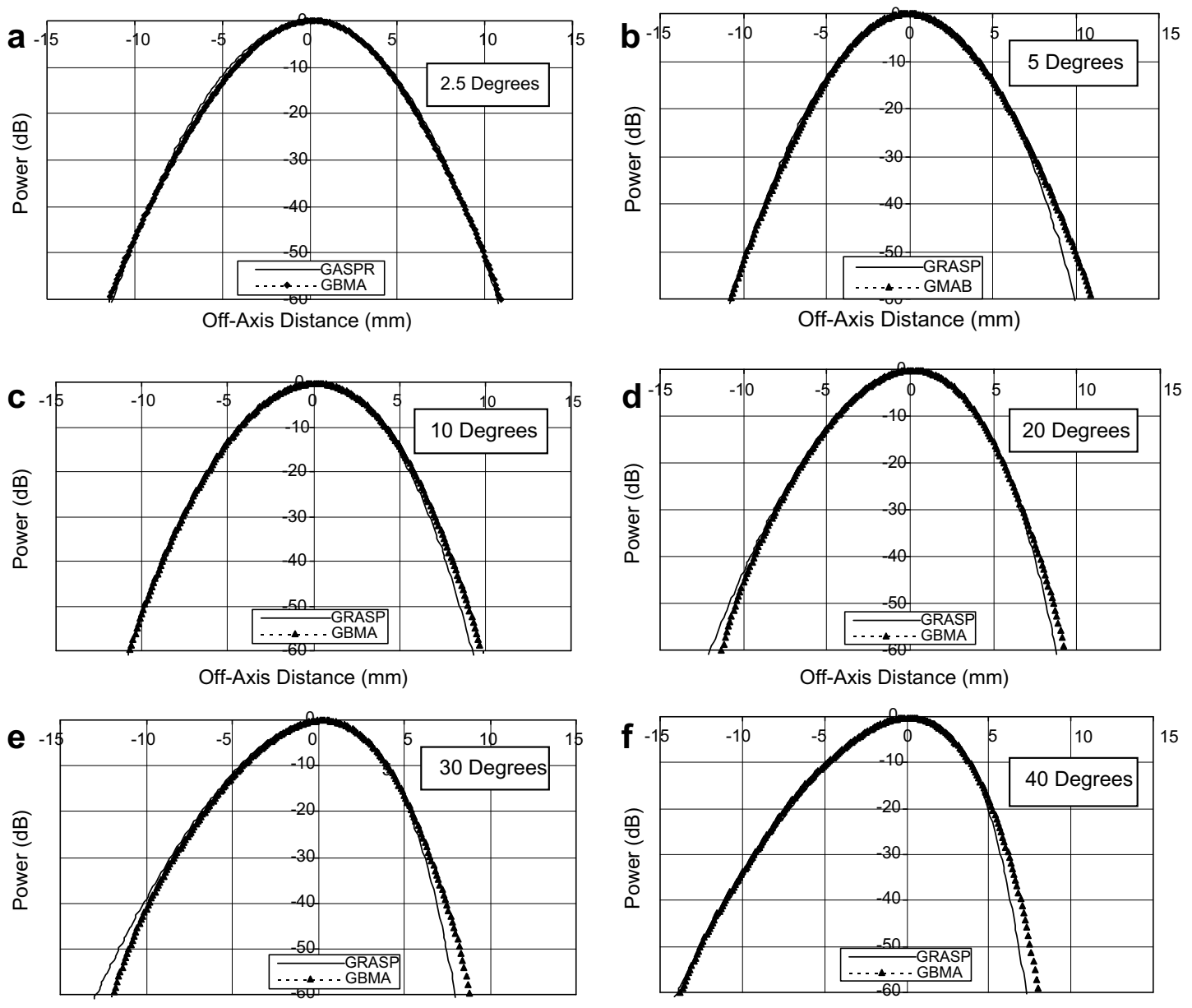

Off-Axis Distance $(\mathrm{mm})$

Off-Axis Distance $(\mathrm{mm})$

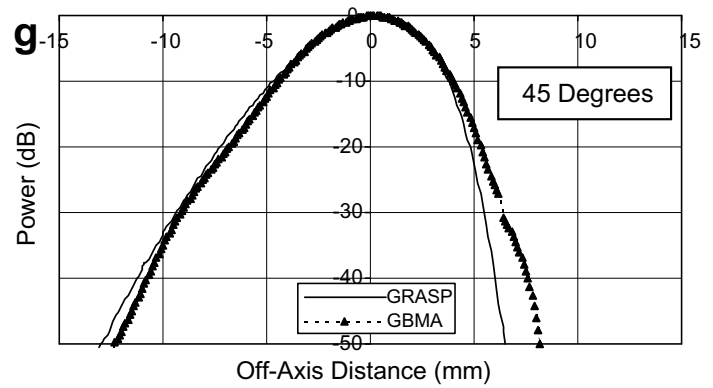

Fig. 5. The amplitude distortion introduced by an off-axis elliptical mirror for increasing angles of incidence.

Table 2

The Gaussicity of the aberrated output field relative to the unaberrated maximum

\begin{tabular}{ll}
\hline Angle of incidence & Gaussicity $(\%)$ \\
\hline Ideal mirror & 100 \\
2.5 & 99.91 \\
5 & 99.88 \\
10 & 99.79 \\
20 & 99.40 \\
30 & 98.68 \\
40 & 97.59 \\
\hline
\end{tabular}

be re-imaged on the second horn. The level and position of the side lobes in the E plane also exhibit good agreement. Additionally the phase shows excellent agreement.

\subsection{Parabolic mirrors}

Parabolic mirror typically finds use in submillimetre systems for transforming a diverging beam to a quasi-collimated beam or vice-versa as illustrated in Fig. 9. In this example the phase centre of the horn is placed at the focus of the parabola, as quasioptically, this is the equivalent of a geometrical point focus. In this GBM scheme the height of 


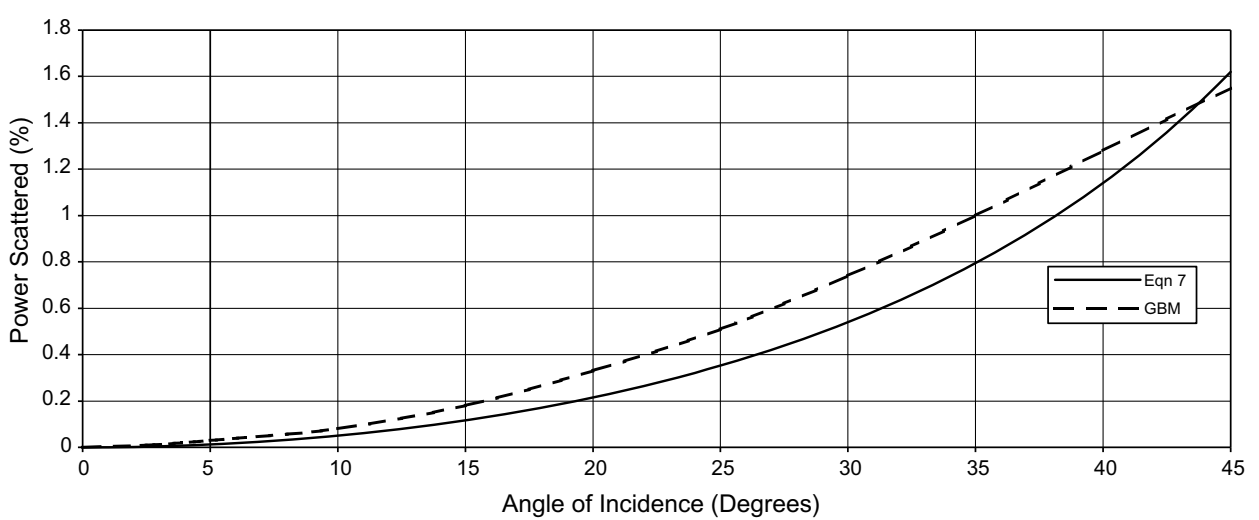

Fig. 6. The relationship of the amount of power removed from the fundamental mode to higher order modes as a function of incidence angle for an offaxis mirror with a fixed beam width and focal length.

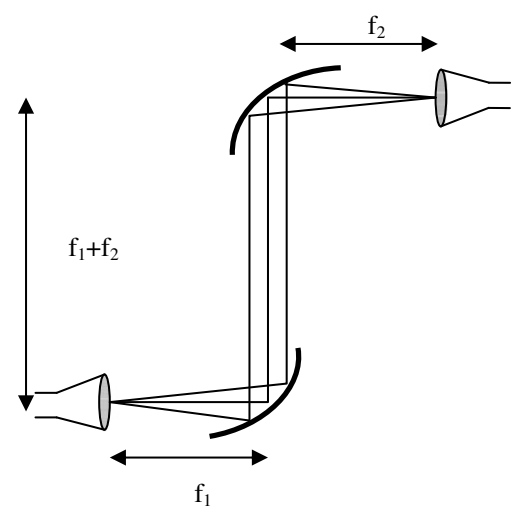

Fig. 7. The compensating 4-f GBT system, consisting of two off-axis elliptical mirrors separated by the sum of their focal lengths.
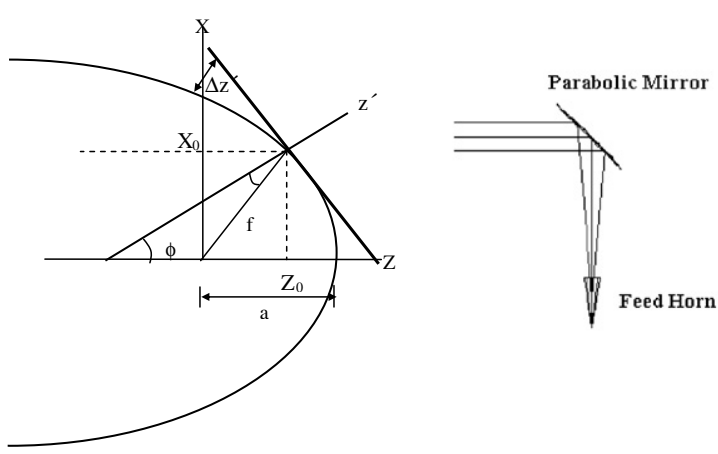

Fig. 9. The geometry of an off-axis parabolic mirror [1] [second plot generated from GRASP GUI].

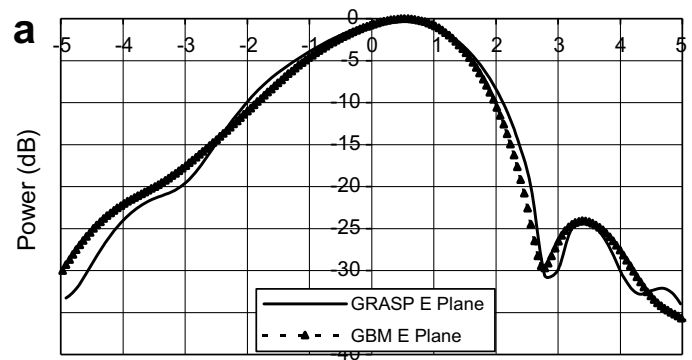

Off-Axis Distance (mm)

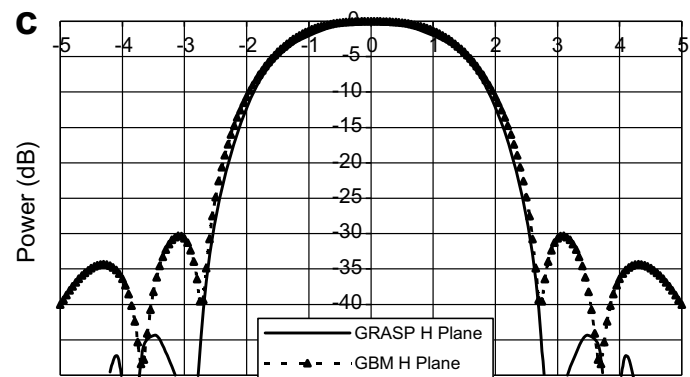

Off-Axis Distance (mm)

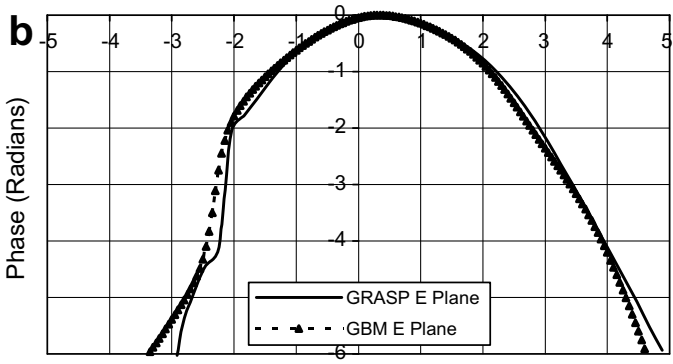

Off-Axis Distance (mm)

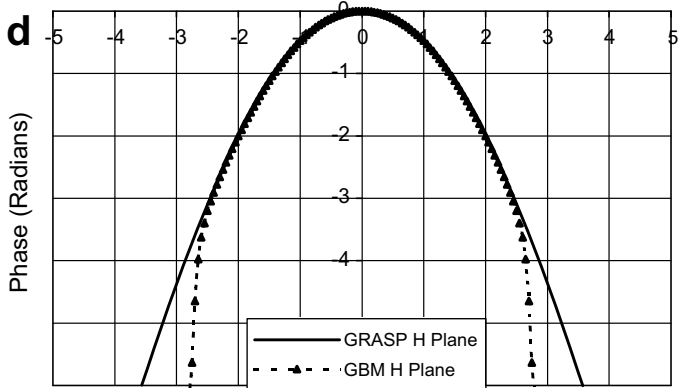

Off-Axis Distance (mm)

Fig. 8. The beam distortion of an off-axis Gaussian beam telescope in the $\mathrm{E}$ and $\mathrm{H}$ plane as predicted by GRASP and GBMA for the power (a, c) and phase $(b, d)$. 
Table 3

The associated parameters for the parabolic mirror

Parabolic mirror input parameters

\begin{tabular}{ll}
\hline Wavelength & $3.19 \mathrm{~mm}$ \\
$W_{\text {in }}$ & $5.11 \mathrm{~mm}$ \\
Input distance & $30 \mathrm{~mm}$ \\
Output distance & $44 \mathrm{~mm}$ \\
Focal length & $30 \mathrm{~mm}$ \\
\hline
\end{tabular}

the parabolic surface over the tangent plane $\Delta z\left(x^{\prime}, y^{\prime}\right)$ must be known and this is again calculated by shifting and rotating the origin of the coordinate frame $(X, Z)$ to the centre of the off-set parabolic such that $\Delta z^{\prime}[1]$

$\Delta z^{\prime}=\left|\frac{-B+\sqrt{B^{2}-4 A C}}{2 A}\right|$,

where the values of $A, B$ and $C$ are related to the input parameters of the parabolic mirror through

$A=\sin ^{2} \delta$,

$B=2 x^{\prime} \sin \delta \cos \delta+2 X_{0} \sin \delta+4 a \cos \delta$,

$C=2 x^{\prime 2} \cos ^{2} \delta+2 x^{\prime} X_{0} \cos \delta-4 a x^{\prime} y^{\prime 2} \sin \delta$.

The input parameters needed to define the mirror is the optical focal length of the mirror $f$. The geometrical focal length of the parent paraboloid is then given by $a=f \cos 2 \delta$ (see Table 3). The coordinates of the centre point of the mirror $X_{0}$ and $Z_{0}$ are given by, respectively, $X_{0}=f \sin 2 \delta$ and $Z_{0}=f \cos 2 \delta$. These parameters are required to calcu- late the $\alpha$ and $\beta$-matrices as described in Eqs. (3) and (4). The input field is a corrugated horn designed to operate at a centre frequency of $94 \mathrm{GHz}$ to illustrate the accuracy of this technique at longer wavelengths than utilised in the previous example. These parameters then have to be calculated for a specific parabolic mirror. For the considered mirror the associated parameters are displayed in Table 3 for an angle of incidence of 45 degrees.

The output distance was chosen to yield the smallest possible beam of $W=5.9 \mathrm{~mm}$. The mirror size was $4 W$. The fields predicted by GRASP and GBMA scatter matrix are compared to determine the accuracy of GBMA in predicting the aberrations introduced by the parabolic mirror. Again 100 modes were used given the accuracy demonstrated by this mode set previously. Fig. 10a-d displays the intensity and phase distribution in both $\mathrm{E}$ and $\mathrm{H}$ planes at a plane $44.1 \mathrm{~mm}$ from the mirror as predicted by the GBM scatter matrix approach which now gives a distorted profile and exhibits good agreement down to $-25 \mathrm{~dB}$ between the GRASP and the scalar GBM approach. Both beams exhibit a squinted beam with an asymmetric beam profile although GBMA predicts a slightly wider beam below $-25 \mathrm{~dB}$, the profile is still well matched and the shift of the peak intensity is the same for both. As with the elliptical mirror there are no distortions introduced in the $\mathrm{H}$ plane as the mirror is only tilted in one direction.

The phase exhibits a distortion in the E plane due to the tilting of the mirror that introduces a phase mismatch across the surface between the incoming fields phase radius

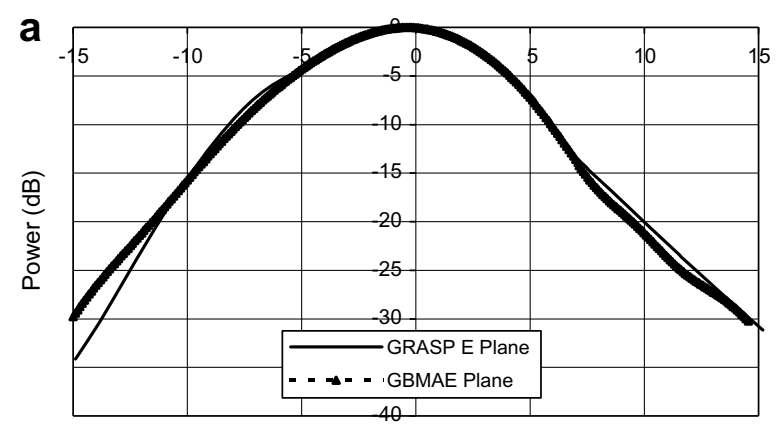

Off-Axis Distance (mm)

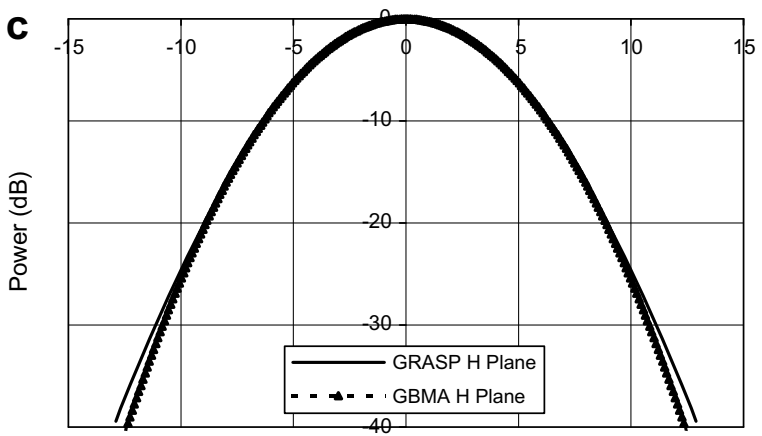

Off-Axis Distance (mm)

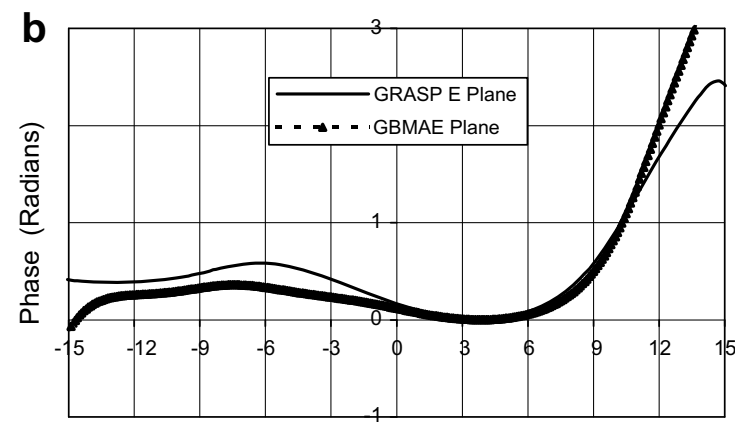

Off-Axis Distance (mm)

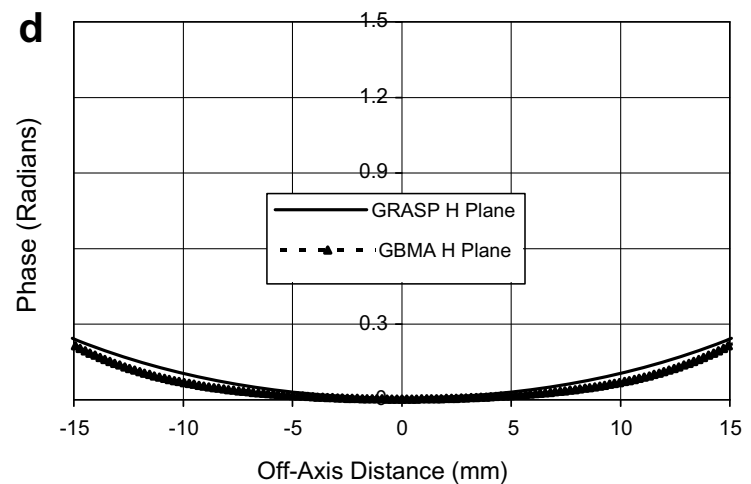

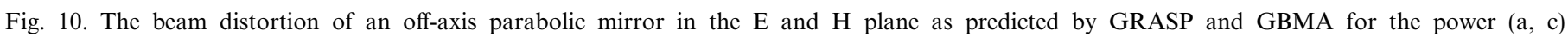
and phase $(b, d)$. 
of curvature and the mirrors curvature. The GBM technique exhibits good agreement in the distorted phase front in the E plane also. As with previous examples the GBMA is limited by its simplifying assumptions but still exhibits excellent agreement with the PO package.

\section{Conclusions}

We have presented here a scalar method based on Gaussian beam modes that allows the calculation of aberrations or distortions induced by off-axis mirrors within the paraxial assumptions inherent to GBMA. Using examples of both elliptical and parabolic surfaces we have validated the theory originally described in [1]. The technique has proven accurate compared to GRASP8 simulations, a physical optics (PO) package for off-axis angles of up to $45^{\circ}$ down to an intensity level of $-25 \mathrm{~dB}$ to $-40 \mathrm{~dB}$ using a limited mode set. The described technique has also been illustrated to represent accurately to levels of $-25 \mathrm{~dB}$ a two-mirror system with a multi-moded input arranged in a compensating configuration. The technique can also predict phase front variation equivalent to that calculated using GRASP8. This GBM technique allows a more intuitive understanding of beam evolution in a quasioptical system than is possible from a rigorous $\mathrm{PO}$ approach, where the basic physical processes can be obscured, and exhibits a similar accuracy.

\section{Acknowledgements}

The authors would like to acknowledge financial support from the Irish Research Council of Science, Engineering and Technology (IRCSET) and from PRODEX through Enterprise Ireland that enabled this work to be completed.

\section{References}

[1] S. Withington, A. Murphy, G. Isaak, Representation of mirrors in beam waveguides as inclined phase transforming surfaces, Infrared Phys. Tech. 36 (3) (1995) 723-734.

[2] P. Goldsmith, Quasi-Optical Systems, IEEE Press Series on Microwave Technology and RF, 1998.

[3] J. Lesurf, "Millimetre Wave Optics", Devices and Systems, AdamHilger and IOP Publishing, 1990.

[4] D. Martin, J. Bowen, Long wave optics, IEEE Trans. Microw. Theory Tech. 41 (10) (1993).

[5] A.D. Olver, Microwave Horns and Feeds, in: IEEE Electromagnetic Waves Series, vol. 39, 1994

[6] J.A. Murphy, N. Trappe, S. Withington, Gaussian beam mode analysis of partial reflections in simple quasioptical systems fed by horn antennas, Infrared Phys. Tech. 44 (2003) 289-297.

[7] N. Trappe et al., Gaussian beam mode analysis of standing waves between two coupled corrugated horns, IEEE Trans. Antenn. Propag. 53 (5) (2005).

[8] A. Siegman, Lasers, University Science Books, 1986.

[9] H. Koelgnik, T. Li, Laser Beams and Resonators, in: Proc IEEE, vol. 54, 1966, pp. 1312-1329. 